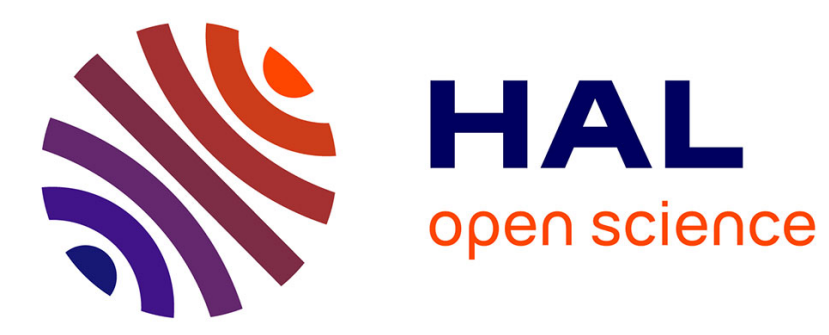

\title{
Ship Identification Based on Ship Blade Noise
}

Haitao Qi, Zhijing Xu

\section{To cite this version:}

Haitao Qi, Zhijing Xu. Ship Identification Based on Ship Blade Noise. 2nd International Conference on Intelligence Science (ICIS), Oct 2017, Shanghai, China. pp.426-434, 10.1007/978-3-319-68121-4_46 . hal-01820917

\section{HAL Id: hal-01820917 https://hal.inria.fr/hal-01820917}

Submitted on 22 Jun 2018

HAL is a multi-disciplinary open access archive for the deposit and dissemination of scientific research documents, whether they are published or not. The documents may come from teaching and research institutions in France or abroad, or from public or private research centers.
L'archive ouverte pluridisciplinaire HAL, est destinée au dépôt et à la diffusion de documents scientifiques de niveau recherche, publiés ou non, émanant des établissements d'enseignement et de recherche français ou étrangers, des laboratoires publics ou privés. 


\title{
Ship Identification based on Ship Blade Noise
}

\author{
Haitao Qi ${ }^{1}$, Zhijing $\mathrm{Xu}^{1}$ \\ College of Information Engineering, Shanghai Maritime University, Shanghai, China \\ $932392658 @ q q . c o m$
}

\begin{abstract}
In this paper, we propose a new method about ship identification, which is based on paddle noise for classification and recognition. Blade noise is the feature that is used to identify ships, blade noise is collected by digital hydrophone, then the signal is converted to spectrum. We extract features based on the difference of the pixel in the picture. What's more, the softmax classification function is used to training blade noise samples by combining with CDBN technology. The maximal output is the sample that satisfies the minimum cost function. The results of our experiment show that the method can be applied for ships identification by combining CDBN, spectrum and softmax functions, the classification recognition rate increases with the increase of the convolution kernel.
\end{abstract}

Keywords: Blade noise; Digital hydrophone; Softmax; CDBN.

\section{Introduction}

In recent years, more and more ship identification methods are forming, because of the development of pattern recognition and artificial intelligence. For example: wavelet analysis theory, support vector machine (SVM)[1], neural network and deep Learning model[2]. Of course, there are other research methods, such as AIS and XAIRCRAFT XCope UAV vision system and Ship identification based on ship blade noise[3].

The traditional ship identification generally uses the theory of wavelet analysis to analyze the characteristics and characteristics of ship noise. The wavelet packet technique is used to separate the characteristics of different frequency bands, and the maximum and outband energy is extracted in the wavelet band. This method is cumbersome and complex, and SVM is valid for small samples. In the neural network of ship identification, the DEMON spectrum is extracted by spectral estimation method, and then BP neural network is used to classify and identify, but the feature extraction can not represent the ship noise well, which is not conducive to the actual operation.

At present, the deep learning technology development is rapid, it can forms a more abstract high-level representation of attribute categories or characteristics by combining low-level features. Convolutional Deep Belief Networks(CDBN)[4] is a method of deep learning, it has been successfully applied in recognition tasks, such as handwritten character recognition, face recognition and pedestrian detection. The experiment of the results show that $\mathrm{CDBN}$ model can extract features effectively. 
In this paper, the noise of different types of ship blades are simulated by setting the number and size of the blades. The traditional neural network and the deep learning model are used respectively. Speedboat model's blades can produce different noise signals and simulate the cavitation noise[5] by using different blades, and then signals are processed by the convolutional belief neural network (CDBN).

\section{Noise Collection of Ship Blade Signal}

Ship radiation noise is mainly composed of propeller noise, mechanical noise and hydrodynamic noise. During the high speed of the ship, the noise generated by the propeller blades is divided into vacuosity and non-voiding noise. Vacuum noise is the main noise in ship sailing.

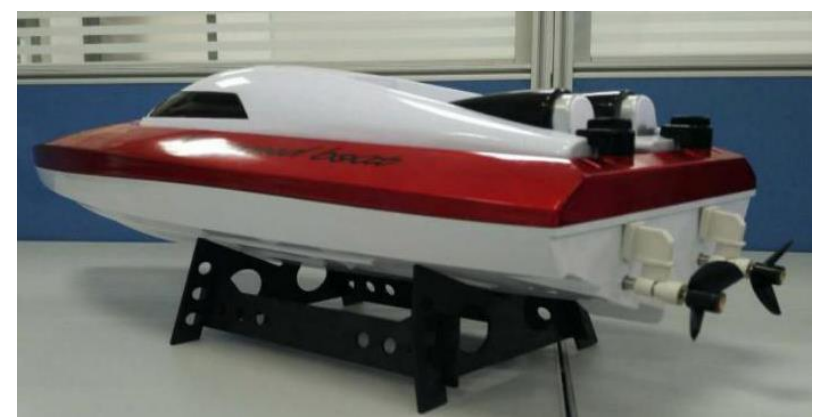

Fig. 1. Speedboat model

As the experimental conditions are limited, this experiment can not collect propeller noise signal, the signals are simulated sampling in still water by a speedboat model, speedboat model is shown in Figure 1. The experiment simulated different ships By changing the type and size of the propeller blades constantly. Noise of the software control interface is shown in Figure 2.

The ship blade noise signal can be divided through the number of blades and the blade size of the propeller. In this experiment, five different blades of the propeller: 2 leaves, 3 leaves, 4 leaves, irregular 4 leaves, irregular 7 leaves. The rules of the ship propeller blades are divided to $24 \mathrm{~mm}, 28 \mathrm{~mm}, 32 \mathrm{~mm}, 36 \mathrm{~mm}, 40 \mathrm{~mm}, 44 \mathrm{~mm}$ according to the size; Irregular 4 leaves and irregular 7 leaves are not required in size. The signals of irregular blades are added to the rules signal to enhance the generalization ability of the deep neural network. Table 1 shows the number of signals collected for different types of propeller blades. 


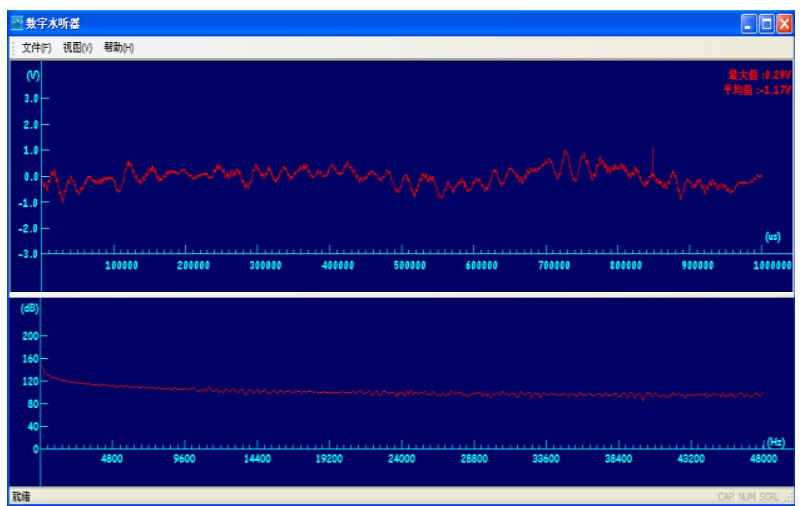

Fig. 2. software control interface

Table 1. Number of different types of blades collected signal

\begin{tabular}{ccccccc}
\hline $\begin{array}{c}\text { Size and } \\
\text { model }\end{array}$ & $\begin{array}{c}24 \\
\mathrm{~mm}\end{array}$ & $\begin{array}{c}28 \\
\mathrm{~mm}\end{array}$ & $\begin{array}{c}32 \\
\mathrm{~mm}\end{array}$ & $\begin{array}{c}36 \\
\mathrm{~mm}\end{array}$ & $\begin{array}{c}40 \\
\mathrm{~mm}\end{array}$ & $\begin{array}{c}44 \\
\mathrm{~mm}\end{array}$ \\
\hline Two blades & 15 & 15 & 15 & 15 & 15 & 15 \\
& 0 & 0 & 0 & 0 & 0 & 0 \\
three blades & 15 & 15 & 15 & 15 & 15 & 15 \\
& 0 & 0 & 0 & 0 & 0 & 0 \\
four blades & 15 & 15 & 15 & 15 & 15 & 15 \\
Irregular four & 0 & 0 & 0 & 0 & 0 & 0 \\
blades & & & & & & \\
Irregular
\end{tabular}

\section{Blade Noise spectrum}

DHP8501 digital hydrophone collects the blade noise signal in the form of binary. Since the binary data is not suitable for filtering and feature extraction, the file format must be converted into a wav format, it is convenient to feature extraction and deep learning, so the binary file is converted to wav format files.

The sound signal is subjected to a windowing function and then subjected to Fourier transform. The resulting amplitude is represented by a spectrum with different shades of color, which is the phonogram of the sound signal we have obtained. In this paper, the blade noise signal is processed in the form of a spectrum similar to the speech signal processed in the form of speech spectrum. From the nature of the spectrum, we can see that the two-dimensional spectrum expresses the three-dimensional characteristics of the sound signal, which is the time characteristic of the horizontal axis, the 
frequency characteristic of the vertical axis and the energy characteristic of the gray scale in the spectrum.

Taking the pulse signal of the speedboat model as an example, this signal collects one of the 20 kinds of blade noise signals of the speedboat model. The number of blades is 4 leaves, the blade size is $32 \mathrm{~mm}$, and the time of blade noise signal is $30 \mathrm{~s}$. The wav format sound signal is represented by a spectrum, as shown in Figure 3.

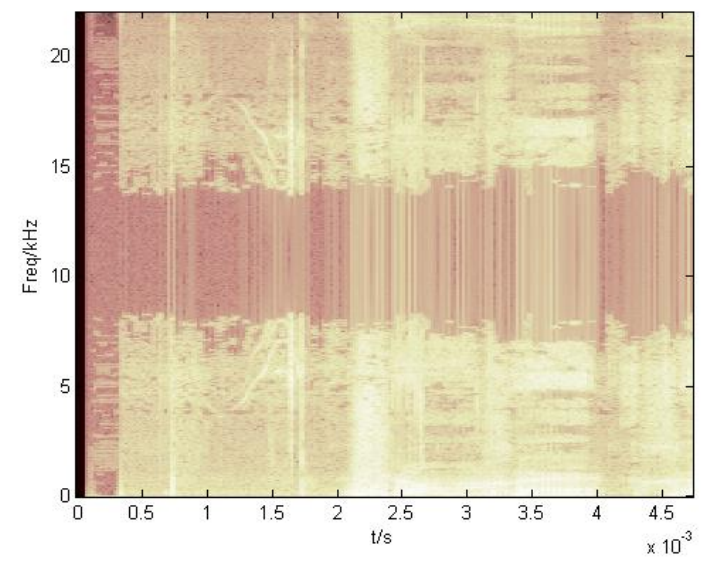

Fig. 3. Impeller noise spectrum

\section{Dimension Reduction and Classification Processing}

\subsection{Probability of the Largest Pool Layer}

In order to solve the problem that the feature value is too large to fitted for the classifier, the pool layer is added to the classifier. Pooling generally uses the method of aggregate statistics to find the characteristic mean for different positions[6]. The pooling process not only reduces the dimensionality of the feature but also enhances the generalization ability of the deep model, which can effectively prevent overfitting.

In the deep learning model[7], there are three types of pooling, which are named average pooling, maximum pooling and random pooling. The average pooling is the average of the numbers in the pooled domain. The maximum pooling is the characteristic point of the neighborhood. The random pool is probability assigns a variable to sampling point and sub-sampled by probability. Convolutional Restricted Boltzmann Machines (CRBM) [8] uses random pooling.

As can be seen from Figure 4, the hidden layer and the pooling layer have the same number of units, it set as $\mathrm{k}$ group according to the mentioned above, the total of pool layer binary is $N_{P} \times N_{P}$. Defining a binary interval $B_{\alpha} \stackrel{\Delta}{=}\{(i, j)\}: h_{i j}$ belong to $\alpha$. The area block $B_{\alpha}$ of the hidden layer is fastened to the cell of the pooled layer. 
Once the hidden layer of cell area block is activated, which is equals to the corresponding cell block is activated.

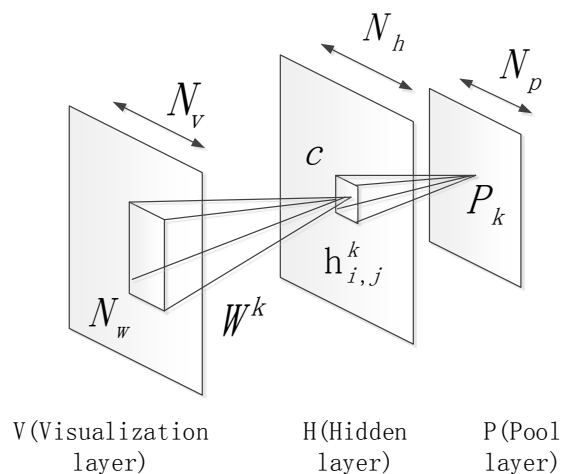

Fig. 4. Added the pool layer to conditional Restricted Boltsman Machine(CRBM)

Then, the maximum pooled CRBM energy function is:

$$
E(v, h)=-\sum_{k} \sum_{i, j}\left(h_{i, j}^{k}\left(\widetilde{W}^{k} *_{V}\right)_{i, j}+b_{k} h_{i, j}^{k}\right)-c \sum_{i, j} V_{i, j}
$$

The corresponding conditional probability is:

$$
\begin{gathered}
P\left(h_{i, j}^{k}=1 \mid V\right)=\frac{\exp \left(I\left(h_{i, j}^{k}\right)\right)}{1+\sum_{\left(i^{\prime}, j^{\prime}\right) \in B_{\alpha}} \exp \left(I\left(h_{i^{\prime}, j^{\prime}}^{k}\right)\right)} \\
P\left(p_{\alpha}^{k}=0 \mid v\right)=\frac{1}{1+\sum_{\left(i^{\prime}, j^{\prime}\right) \in B_{\alpha}} \exp \left(I\left(h_{i^{\prime}, j^{\prime}}^{k}\right)\right)}
\end{gathered}
$$

In the above equation, $-I\left(h_{i, j}^{k}\right)$ is the energy increment expression of the activation function, the form follow as:

$$
I\left(h_{i, j}^{k}\right) \stackrel{\Delta}{=} b_{k}+\left(\widetilde{W}^{k} * v\right)_{i, j}
$$

\subsection{Softmax Classification Function}

The softmax classifier is a logistic regression function classifier that can be classified as multiple categories, which is a commonly used classifier for the deep learning classification model. The basic principle is to calculate the probability value of all independent samples, the probability value which meets the minimum sample regard as the optimal sample output, so as to achieve the effect of classification. Sigmoid is the activation function of the softmax classifier:

$$
g(z)=\frac{1}{1+e^{-z}}
$$


the way of mathematical description is the same as below. Supposing there are $\mathrm{m}$ training sample $:\left\{\left(\mathrm{x}^{(1)}, \mathrm{y}^{(1)}\right), \ldots,\left(\mathrm{x}^{(m)}, \mathrm{y}^{(m)}\right)\right\}$ For sample $\mathrm{x}$, there is always a corresponding $\mathrm{k}$-dimensional vector $\mathrm{y}$ as classification label. Then for $\left\{\left(\mathrm{x}^{(1)}, \mathrm{y}^{(1)}\right), \ldots,\left(\mathrm{x}^{(m)}, \mathrm{y}^{(m)}\right)\right\}$ with $\mathrm{y}^{(\mathrm{m})} \in\{1,2, \ldots, k\}$, the dimension of $x$ is $\mathrm{n}+1, \theta=\left\{\mathrm{W}_{\mathrm{ij}}, \mathrm{b}_{\mathrm{j}}\right\}$, then the probability that the class is judged to be $\mathrm{k}$ in the input is $\mathrm{x}$ is the $p(y=j \mid x)$, and the probability is chosen among all the probabilities. The category is the optimal solution. The $\mathrm{K}$ input classifier will get a k-dimensional output vector, and the sum of all the elements of the vector is 1 . Output set to:

$$
h_{\theta}\left(x^{(i)}\right)=\left(\begin{array}{c}
p\left(y^{(i)}=1 \mid x^{(i)} ; \theta\right) \\
p\left(y^{(i)}=2 \mid x^{(i)} ; \theta\right) \\
\vdots \\
p\left(y^{(i)}=k \mid x^{(i)} ; \theta\right)
\end{array}\right)=\frac{1}{Z}\left(\begin{array}{c}
\exp \left(\theta_{1}^{T}\left(x^{(i)}\right)\right) \\
\exp \left(\theta_{2}^{T}\left(x^{(i)}\right)\right) \\
\vdots \\
\exp \left(\theta_{k}^{T}\left(x^{(i)}\right)\right)
\end{array}\right)
$$

Where the model parameter is $\theta_{1}, \theta_{2}, \cdots \theta_{k}$ and the normalized function is $Z=\sum_{j=1}^{k} \exp \left(\theta_{1}^{T}\left(x^{(i)}\right)\right)$. Now solving the probabilistic problem is trans-

formed into solving the model parameters. In order to solve the model function, the cost function of the regression model is introduced. If the parameter makes the value of the cost function reach the minimum, the parameter is the solution. The cost function is set to:

$$
J(\theta)-\frac{1}{m} \sum_{i=1}^{m} \sum_{j=1}^{k} 1\left\{y^{(i)}=j\right\} \lg \frac{\exp \left(\theta_{1}^{T}\left(x^{(i)}\right)\right)}{\sum_{j=1}^{k} \exp \left(\theta_{1}^{T}\left(x^{(i)}\right)\right)}
$$

For solving the cost function, the usual method is to find the partial derivative of the cost function, that is $j(\theta)$ for each parameter $\theta_{j}$ partial derivative, so that the partial derivative is 0 , solving its value, and reversing to solve the sample parameter probability value.

\section{Experimental Setup and Analysis of Results}

\subsection{Spectral Classification and Recognition Model}

Using the web tutorial to produce the generated blade noise map into a data set similar to CIFAR-10.

Taking 30,000 samples from the data set and dividing the sample into two cases:

In first case, 10,000 samples will be divided into two groups, one group selected 8,000 samples as a training sample, leaving the 2000 samples as a test sample.

In second case, 20,000 samples will be divided into two groups, one group selected 16,000 samples as a training sample, leaving 4000 samples as a test sample.

First, preprocessed the data set: the first step is to whiten the data, reduce the correlation of adjacent pixels, so as to reduce the correlation between the features, and 
between the characteristics has the same variance. The second step, the data is divided into several copies, the number of each sample is 100. In this experiment, the CDBN structure is set as: the input layer is $32 \times 32 \times 3$; the convolution layer is $7 \times 7 \times 10$; the pool layer is $2 \times 2 \times 2$; and the second layer structure is set as: the convolution layer is $7 \times 7 \times 12$; the Pool layer is $2 \times 2 \times 2$. In this experiment, the number of layers and convolution kernel, and the size of the convolution kernel are not specifically prescribed.

\subsection{Classification and Recognition on Samples}

In this experiment, the classification recognition rate was tested separately, in the first case tested by 10,000 samples, in the second case tested by 20,000 samples. The network structure of $\mathrm{CDBN}$ is set as: the input layer is $32 \times 32 \times 3$; the convolution layer is $7 \times 7 \times 10$; the pool layer is $2 \times 2 \times 2$; and the second layer structure is set as: the convolution layer is $7 \times 7 \times 12$; the pool layer is $2 \times 2 \times 2$. The specific parameters of the network are: learning rate is 0.05 , the number of iterations is 100 times. According to the above data to get the spectrum CDBN model classification recognition rate as shown in Table 2:

Table 2. The number of samples and the impact of ICA and ASSASE on the classification recognition rate

\begin{tabular}{ccc}
\hline The way & $\begin{array}{l}\text { The first } \\
\text { case }\end{array}$ & The second case \\
\hline $\begin{array}{c}\text { Classification } \\
\text { recognition rate }\end{array}$ & $65.2 \%$ & $68.2 \%$ \\
\hline
\end{tabular}

As can be seen from the table, in general, the sample classification recognition rate is not very high, and the number of samples is high and the classification rate of the model is higher.

\subsection{Recognition Rate about CDBN Network Structure}

Since the recognition rate of the spectrum increases with the increase of the test sample, so in the test CDBN network structure on the impact of the impact of the experiment, only the second group of samples were tested. In this experiment, the experimental data are set as follows: The experiment divided 30,000 samples into two groups, 22,500 as training samples, and 7,500 as test samples. CDBN is a single layer structure, the input layer is $32 \times 32 \times 3$, the pool layer is $2 \times 2 \times 2$. Learning rate is 0.05 , the number of iterations are 200 times. The experimental results are shown in Figure 5 and Table 3. 


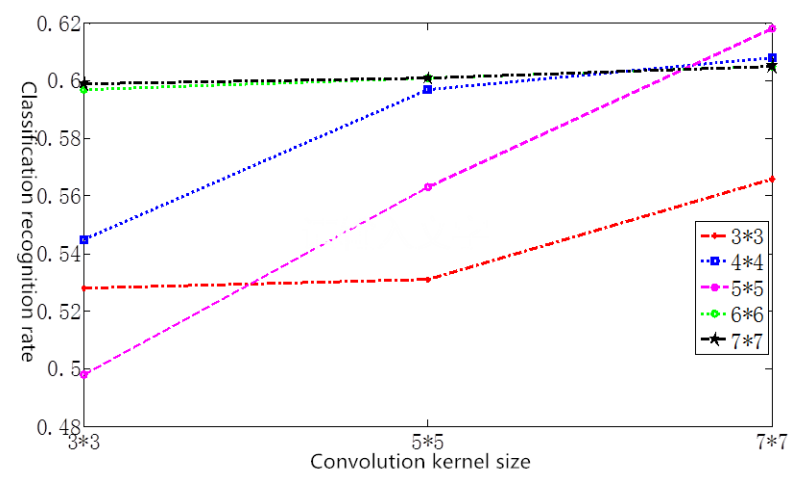

Fig. 5. Influence of Network Structure on Classification Recognition Rate

Table 3. The Influence of CDBN Network Structure on Classification Recognition Rate

\begin{tabular}{ccc}
\hline \multicolumn{3}{c}{ Single layer structure : A layer of convolution +A layer of pooling } \\
\hline \multirow{2}{*}{ Convolution count } & Convolution kernel & Classification recognition rate \\
\hline \multirow{2}{*}{3} & $3 \times 3$ & $52.8 \%$ \\
& $5 \times 5$ & $52.9 \%$ \\
& $7 \times 7$ & $56.0 \%$ \\
& $3 \times 3$ & $54.6 \%$ \\
4 & $5 \times 5$ & $59.6 \%$ \\
& $7 \times 7$ & $60.3 \%$ \\
& $3 \times 3$ & $49.2 \%$ \\
5 & $5 \times 5$ & $55.6 \%$ \\
& $7 \times 7$ & $61.8 \%$ \\
& $3 \times 3$ & $59.8 \%$ \\
6 & $5 \times 5$ & $60.0 \%$ \\
& $7 \times 7$ & $60.3 \%$ \\
& $3 \times 3$ & $60.0 \%$ \\
& $5 \times 5$ & $60.3 \%$ \\
& $7 \times 7$ & $60.7 \%$ \\
\hline
\end{tabular}

It can be seen from Figure 5 that as the number of convolution nuclei is from less to more, the classification recognition is increased firstly, and the classification recognition rate is almost constant at a certain stage. In the case of a certain number, the classification recognition rate is higher. The classification of the kernel increases the 
classification rate, but the classification recognition rate does not fluctuate in the case of a large number.

\section{Conclusions}

In this paper, we provide a new method of ship identification, which is based on CDBN technology to carry out ship classification and identification. In the experiment, we demonstrated the possibility of CDBN, spectrum and softmax to identify ships. Experimental results on identification rate of ship show that recognition rate of ship will increase when increasing the samples of the training; the number of convolution kernel is different, the recognition rate is different, too. Correspondingly, when we increase the number of convolution kernel, the recognition rate will increase. Therefore, if we want to improve the recognition rate of ship, we must increase the number of training samples and convolution kernel.

Acknowledgments. This study was funded by the National Natural Science Foundation of China (Grant No.61404083).

\section{References}

1. Suykens J A K, Vandewalle J. Least squares support vector machine classifiers[J]. Neural processing letters, 1999, 9(3): 293-300.

2. Tang Y. Deep learning using linear support vector machines[J]. arXiv preprint arXiv:1306.0239, 2013.

3. Can G, Akbaş C E, Çetin A E. Recognition of vessel acoustic signatures using non-linear teager energy based features[C]//Computational Intelligence for Multimedia Understanding (IWCIM), 2016 International Workshop on. IEEE, 2016: $1-5$.

4. Lee H, Pham P, Largman Y, et al. Unsupervised feature learning for audio classification using convolutional deep belief networks[C]//Advances in neural information processing systems. 2009: 1096-1104.

5. Harrison M. An experimental study of single bubble cavitation noise[J]. The Journal of the Acoustical Society of America, 1952, 24(6): 776-782.

6. Adhikari S P, Yang C, Kim H, et al. Construction of CNN Template via Learning with Random Weight Change Algorithm[J]. CNNA 2016, 2016.

7. Sainath T N, Kingsbury B, Saon G, et al. Deep convolutional neural networks for large-scale speech tasks[J]. Neural Networks, 2015, 64: 39-48.

8. Krizhevsky A, Hinton G E. Factored 3-way restricted boltzmann machines for modeling natural images[C]//International conference on artificial intelligence and statistics. 2010: 621-628. 